\title{
Simplified Nonlinear Modeling of River Flow Routing
}

\author{
Xavier Litrico*,a ${ }^{*}$ Jean-Baptiste Pomet $^{\mathrm{b}}$, Vincent Guinot ${ }^{\mathrm{c}}$ \\ ${ }^{a}$ Cemagref, UMR G-EAU, 361 rue JF Breton, B.P. 5095, 34196 Montpellier Cedex 5, \\ France. Tel: +33467046347. Fax: +33467166440. \\ ${ }^{b}$ INRIA, Projet APICS, B.P. 93, 06902 Sophia Antipolis Cedex, France. Tel: +33 4 92 \\ 3877 79. Fax: +33492387858. \\ ${ }^{c}$ Hydrosciences Montpellier UMR 5569 (CNRS, IRD, UM1, UM2), France. Tel: +334 \\ 671490 56. Fax: +33467144774.
}

\begin{abstract}
Management of open-channel flow systems requires accurate models of flow transfer. This article presents a simple nonlinear model representative of the flow transfer in a river reach. The model is obtained through linearization of a physical model, simplification using the cumulant matching method and analytic identification of a nonlinear model coinciding with the linear model around equilibrium points, corresponding to the hydraulic permanent regimes. The methodology is illustrated on the diffusive wave equation and the Saint-Venant equations. The obtained nonlinear models are compared in simulation to the initial models. The nonlinear model is shown to ensure mass conservation, despite the variable delay element of the model. The proposed model can reproduce the nonlinear behavior of the time-delay with discharge variations. It is well-suited for fast simulations, flow forecasting, and for controller design.
\end{abstract}

Key words:

Open-channel hydraulic system, Flow routing, Nonlinear system, Water Flow Modeling, Variable time delay

\footnotetext{
*Corresponding author

Email addresses: xavier.litrico@cemagref.fr (Xavier Litrico), pomet@sophia.inria.fr (Jean-Baptiste Pomet), guinot@msem.univ-montp2.fr (Vincent Guinot)
} 


\section{Introduction}

The management of a watershed usually relies on accurate modeling for forecasting purposes, or for operational management. However, in choosing such models, there is a classical trade-off between simplicity and precision in terms of time delay, diffusion and volume transited. Complex models are difficult to use for real-time management, and they require long computational time, while simplified models are less accurate but more amenable to be used either for management purposes, operational control, or quick forecasting.

Complex models have the advantage of accuracy, provided parameters are correctly tuned; given the uncertainty of data used for identification, huge models clearly have to be avoided for they generate identification problems following from over-parametrization [7]. It is also interesting to use parameters with a physical meaning, to have an additional validation of identification results. A simple model requires less computational time than a complex one and can therefore more easily be used for real-time forecasting and managing purposes.

This is why a lot of research effort has been spent into finding appropriate simplified models for flow routing [27]. Different linear models, such as the Hayami model [13, 24], or Muskingum model [10], or stock models have been developed. Their main limitation is their narrow domain of validity; used to represent a nonlinear phenomenon, these linear models are only valid if the discharge remains near to the point of linearization.

To alleviate this point, some simplified nonlinear models were developed to replicate the nonlinear feature of flow transfer in a reach [5]. However, these models do not accurately represent the time delay variation, which is an important characteristic of the flow transfer for low discharges.

A multilinear approach was used by [8] to develop a model for flow routing that reproduces the nonlinear variation of the time delay with the flow. Unfortunately, as shown by [26], their multilinear discrete lag-cascade model can sometimes present abrupt falls or discontinuities in the routed hydrograph. As a remedy to this deficiency, [26] proposed to constrain the lag to a fixed value, but this also removes most of the interest of the multilinear model, since in that case the delay does not change with the flow. The model is then closer to the quadratic lag and route model proposed in [5], which has a fixed delay.

The objective of the present article is to obtain a simple nonlinear model for the discharge transfer in a reach from a knowledge-based model, in order 
to keep parameters with a physical meaning. In particular, this model should be adapted to the case of rivers with low flows, where the reproduction of the variations of the delay according to the discharge is required. We analyze the nonlinear model, and give conditions under which it is conservative and does not present discontinuities in the routed hydrograph.

In more mathematical terms, the proposed model is obtained following a methodology to reduce a nonlinear Partial Differential Equation (PDE) to a nonlinear Delay Differential Equation (DDE):

1. First the nonlinear PDE is linearized around a steady flow discharge,

2. The Laplace transform is used to derive the inflow/outflow transfer function,

3. The cumulant matching method is used to approximate this transfer function by the transfer function of a Delay Differential Equation (here a first order transfer function with delay),

4. We then derive a nonlinear DDE which coincides with the linear DDE for every steady flow discharge.

The proposed methodology can be applied to any flow transfer model, since the key element in the proof is that the linearized system should only depend on a scalar variable, here the steady flow discharge. The methodology is here presented, first for the diffusive wave equation, which is a parabolic PDE, and then for the Saint-Venant equations, which are hyperbolic equations. The simplified model is in both cases a nonlinear first order model with delay. The method is nonetheless detailed for the general case of a finite order model with delay.

\section{Proposed Methodology for Simplified Nonlinear Modeling of River Flow Routing}

\subsection{Obtaining a Family of Linear DDEs from a Nonlinear PDE}

In this section, we develop the first three steps of the proposed methodology. We first develop the methodology for the diffusive wave equation. However, the proposed methodology can be applied to any flow model, provided the nonlinearity can be linked to a scalar variable. In most applications, the base flow can be used for this purpose. To show that our approach can be used with other flow models, we also study the Saint-Venant equations in a subsequent section. 


\subsubsection{The Nonlinear PDE: Diffusive Wave Model}

The diffusive wave equation is a partial differential equation obtained by simplification of Saint-Venant model [18]:

$$
\frac{\partial Q}{\partial t}+\Theta(Q) \frac{\partial Q}{\partial x}-E(Q) \frac{\partial^{2} Q}{\partial x^{2}}=0
$$

with $x$ the abscissa, $Q(x, t)$ the discharge $\left(\mathrm{m}^{3} / \mathrm{s}\right), \Theta(Q)$ the celerity $(\mathrm{m} / \mathrm{s})$ and $E(Q)$ the diffusion coefficient $\left(\mathrm{m}^{2} / \mathrm{s}\right)$.

The boundary conditions are given by $Q(0, t)=u(t), \lim _{x \rightarrow \infty} \frac{\partial Q(x, t)}{\partial x}=0$. The output is $w(t)=Q(L, t)$, where $L$ is the length of the river stretch.

$\Theta(Q)$ and $E(Q)$ have relatively complex expressions in the general case, but simpler expressions are available for specific river geometry. Assuming a uniform geometry, and neglecting the effect of backwater curves, $\Theta$ and $E$ can be expressed as functions of the discharge $Q$. In particular for a wide, rectangular channel (slope $S_{b}$, width $W$, Manning friction coefficient $n$ ), $\Theta$ and $E$ are given by:

$$
\begin{aligned}
\Theta(Q) & =\frac{5 S_{b}^{0.3} Q^{0.4}}{3 W^{0.4} n^{0.6}} \\
E(Q) & =\frac{Q}{2 W S_{b}}
\end{aligned}
$$

The above expressions can be replaced by more complicated functions of $Q$ (for example functions obtained by identification in case of a more realistic geometry [15]), without modifying the method exposed below.

\subsubsection{The Linear PDE: Hayami Model}

Linearizing equation (1) around a constant reference discharge $Q_{0} \neq 0$ $\left(Q=Q_{0}+q\right)$ gives the Hayami equation:

$$
\frac{\partial q}{\partial t}+\Theta_{0} \frac{\partial q}{\partial x}-E_{0} \frac{\partial^{2} q}{\partial x^{2}}=0
$$

with $\Theta_{0}=\Theta\left(Q_{0}\right)$ and $E_{0}=E\left(Q_{0}\right)$.

Considering the boundary conditions $u(t)=q(0, t)$ and $\lim _{x \rightarrow \infty} \frac{\partial q}{\partial x}=0$, and the measured output $w(t)=q(L, t)$, the linear PDE (4) can be represented by an irrational transfer function in the Laplace domain (see Appendix A for details), relating upstream and downstream discharge variations:

$$
\hat{w}(s)=F_{\text {Hayami }}(s) \hat{u}(s),
$$


where $\hat{f}(s)$ denotes the Laplace transform of function $f(t), s$ is the Laplace variable $\left(\mathrm{s}^{-1}\right)$, and $F_{\text {Hayami }}(s)$ is the Hayami transfer function:

$$
F_{\text {Hayami }}(s)=e^{\frac{\Theta_{0}-\sqrt{\Theta_{0}^{2}+4 E_{0} s}}{2 E_{0}} L} .
$$

\subsubsection{Approximation of the Linear PDE by a Linear DDE}

The flow routing in a river reach can be approximated by a rational model of first order with delay:

$$
\begin{aligned}
& K \dot{v}(t)+v(t)=u(t) \\
& w(t)=G v(t-\tau)
\end{aligned}
$$

where $G$ is the gain, $K$ is the first order time constant, $\tau$ the time delay, and $v$ is the state of the system.

However, it is not so easy to compute correct values for $G, K$ and $\tau$ so that the output of the rational model fits the one of the Hayami model. One way to do this is to use the cumulant matching method [14].

Cumulant Matching Method. Consider a transfer function $\hat{f}(s)$. The TaylorLagrange development of $\log (\hat{f}(s))$ around $s=0$ at order 2 reads:

$$
\log (\hat{f}(s))=M_{0}-M_{1} s+M_{2} \frac{s^{2}}{2}+o(s)^{2},
$$

where $o(s)$ is a function of $s$ such that $\lim _{s \rightarrow 0} \frac{o(s)}{s}=0$, and the functions $M_{n}$

$$
M_{n}=(-1)^{n} \frac{\mathrm{d}^{n} \log (\hat{f}(s))}{\mathrm{d} s^{n}}(0) .
$$

are the cumulants of $\hat{f}(s)$.

The cumulant matching method [14] provides a rationale for reproducing the behavior of a given transfer function in the low frequency range (obviously the only one that matters in natural systems of our kind), by equating the low order cumulants of two different models and, for instance, construct a rational model that has the same ones as a given irrational transfer function.

Application to the Hayami Transfer Function. In the following, we use the cumulant matching method to fit the frequency response of the Hayami transfer function (5) to that of the first order model with delay (6), which possesses the following transfer function:

$$
F_{1 r}(s)=\frac{G e^{-s \tau}}{1+K s}
$$


Equating the three cumulants of the two models leads to:

$$
\begin{aligned}
\log G & =0 \\
\tau+K & =\frac{L}{\Theta_{0}} \\
K^{2} & =\frac{2 L E_{0}}{\Theta_{0}^{3}}
\end{aligned}
$$

Solving for the unknowns $G, K$ and $\tau$ leads to:

$$
\begin{aligned}
G & =1, \\
\tau & =\frac{L}{\Theta_{0}}-K, \\
K & =\sqrt{\frac{2 L E_{0}}{\Theta_{0}^{3}}} .
\end{aligned}
$$

This identification is valid as long as $\tau>0$, which is equivalent to $L \Theta_{0} /\left(2 E_{0}\right)>1$, or $\chi=3 L \Theta_{0} /\left(10 E_{0}\right)>0.6$, where $\chi$ is a dimensionless coefficient first introduced by [4] to characterize the dynamics of open-channel flow. It is also possible to extend the application of the model in cases when $\chi \leq 0.6$, by forcing the time constant $K$ to zero. In that case, we have a pure delay system with $\tau=L / \Theta_{0}$.

For larger values of dimensionless parameter $\chi$, it is possible to identify a second order model with delay (see e.g. [22]). The obtained DDE model is then a better approximation of the original PDE. However, the second order model may become unstable if $\chi$ becomes smaller than 1.35, which may occur for large values of $Q_{0}$. Therefore, we develop in the article the case of a first order model with delay, which is often sufficient for practical purposes. The proposed methodology can nonetheless be applied on larger order models such as the second order model with delay (see [21]).

\subsection{Obtaining a Nonlinear DDE from a Family of Linear DDEs}

We are now in the situation where we have a family of linear models, indexed by the value $Q_{0}$ of the (constant) discharge around which they are valid. A natural question to ask is whether it is possible to find a single nonlinear system that would have these linear systems as linear approximations at a suitable family of operating points. 
The problem can be posed slightly more generally as follows. We consider a family of linear delay differential systems $\left(\Sigma \mathcal{L}_{\lambda}\right)_{\lambda \in \mathbb{R}}$ indexed by a scalar parameter $\lambda$ :

$$
\Sigma \mathcal{L}_{\lambda}\left\{\begin{aligned}
\dot{v}(t) & =\mathbf{A}(\lambda) v(t)+\mathbf{B}(\lambda) u(t) \\
w(t) & =\mathbf{C}(\lambda) v(t-\tau(\lambda))
\end{aligned}\right.
$$

where $\mathbf{A} \in \mathbb{R}^{n \times n}, \mathbf{B} \in \mathbb{R}^{n \times n_{u}}$ and $\mathbf{C} \in \mathbb{R}^{n_{y} \times n}$ are real matrices, and $v(t) \in \mathbb{R}^{n}$ is the state of the system. $n, n_{u}$ and $n_{y}$ are integers denoting respectively the state dimension, the input dimension and the output dimension.

The question at hand now becomes: Given the family of linear systems (16), does there exist a nonlinear system

$$
\begin{aligned}
\dot{\nu}(t) & =f(\nu(t), u(t)) \\
\omega(t) & =h(\nu(t-\sigma(\nu))),
\end{aligned}
$$

and a curve $\lambda \mapsto \nu_{0}^{\lambda}$ of equilibria, i.e.

$$
f\left(\nu_{0}^{\lambda}, u_{0}^{\lambda}\right)=0
$$

such that each system $\Sigma \mathcal{L}_{\lambda}$ be the linearized system of (17) around $\left(\nu_{0}^{\lambda}, u_{0}^{\lambda}\right)$ ?

The following lemma expresses the linearization of the delay differential system (17) around an equilibrium point, i.e., around $(\bar{\nu}, \bar{u})$ with $f(\bar{\nu}, \bar{u})=0$.

Lemma 1. Consider an equilibrium point $f(\bar{\nu}, \bar{u})=0$ and the stable time invariant linear system with delay

$$
\begin{aligned}
\delta \dot{\nu}(t) & =\mathbf{A} \delta \nu(t)+\mathbf{B} \delta u(t) \\
\delta \omega(t) & =\mathbf{C} \delta \nu(t-\sigma(\bar{\nu})),
\end{aligned}
$$

with

$$
\mathbf{A}=\frac{\partial f}{\partial v}(\bar{\nu}, \bar{u}), \quad \mathbf{B}=\frac{\partial f}{\partial u}(\bar{\nu}, \bar{u}), \quad \mathbf{C}=\frac{\partial h}{\partial v}(\bar{\nu}) .
$$

Then the output $\omega(t)$ of the nonlinear system (17) starting at $\nu(0)=\bar{\nu}$ with input $t \mapsto \bar{u}+\delta u(t)$ is given by

$$
\omega(t)=h(\bar{\nu})+\delta \omega(t)+\varepsilon(\delta \nu, \delta u),
$$

where $\delta \omega(t)$ is the output of the linear system (19) starting at $\delta \nu(0)=0$ with input $\delta u(t)$, and where $\varepsilon$ vanishes at order 1 with respect to its two arguments. 
Applying Lemma 1 to the above question, we can find a nonlinear system (17) if we solve the following relations for $f, h$ and $\left(\nu_{0}^{\lambda}, u_{0}^{\lambda}\right)$

$$
\begin{aligned}
\mathbf{A}(\lambda) & =\frac{\partial f}{\partial v}\left(\nu_{0}^{\lambda}, u_{0}^{\lambda}\right), \\
\mathbf{B}(\lambda) & =\frac{\partial f}{\partial u}\left(\nu_{0}^{\lambda}, u_{0}^{\lambda}\right), \\
\mathbf{C}(\lambda) & =\frac{\partial h}{\partial v}\left(\nu_{0}^{\lambda}\right), \\
\tau(\lambda) & =\sigma\left(\nu_{0}^{\lambda}\right),
\end{aligned}
$$

with the additional constraint (18).

It is clear that there are infinitely many solutions, i.e. functions $f, h$, $\sigma$ and $\left(\nu_{0}^{\lambda}, u_{0}^{\lambda}\right)$ satisfying these relations in particular because one has constraints on $f, h$ and $\sigma$ only along the curve $\lambda \mapsto\left(\nu_{0}^{\lambda}, u_{0}^{\lambda}\right)$.

Basically, one may choose a curve $\lambda \mapsto\left(\nu_{0}^{\lambda}, u_{0}^{\lambda}\right)$ such that

$$
\mathbf{A}(\lambda) \frac{\partial \nu_{0}^{\lambda}}{\partial \lambda}+\mathbf{B}(\lambda) \frac{\partial u_{0}^{\lambda}}{\partial \lambda}=0
$$

and then choose for $f$ a function that is zero along this curve and has the prescribed Jacobian at points on this curve. Any continuous extension away from the curve that satisfies this first order initial data is valid. $h$ is defined up to an additive constant on that curve and can be continuously extended in the same way.

In the sequel, the curve is a straight line and we choose a quasi-linear model as the most natural continuous extension of the linear system.

\subsection{Application to the Family of Linear Models Obtained in Section 2.1.3}

The model obtained by the cumulant matching method from the model of Hayami has parameters that depend explicitly on the reference discharge $Q_{0}$, since $\Theta_{0}$ and $E_{0}$ depend on $Q_{0}$. We can therefore apply the results of section 2.2 .

The flow transfer in the river is now represented by a first order transfer function with delay $F_{1 r}(s)=\frac{e^{-\tau s}}{1+K s}$ with $\tau$ and $K$ given by the equations (11-12). Choosing $\delta v=\delta y$ as a state variable for the system without delay, this linear system can be represented in state space by:

$$
\left\{\begin{array}{l}
\dot{\delta v}(t)=\mathbf{A}(\lambda) \delta v(t)+\mathbf{B}(\lambda) \delta u(t) \\
\delta w(t)=\mathbf{C}(\lambda) \delta v(t-\tau(\lambda))
\end{array}\right.
$$


with $\lambda=Q_{0}, \nu_{0}^{\lambda}=\lambda, u_{0}^{\lambda}=\lambda, \mathbf{A}(\lambda)=-\frac{1}{K(\lambda)}, \mathbf{B}(\lambda)=\frac{1}{K(\lambda)}, \mathbf{C}(\lambda)=1$.

Consider now the nonlinear system described by

$$
\left\{\begin{array}{l}
\dot{v}(t)=\frac{1}{K(v(t))}(u(t)-v(t)) \\
w(t)=v(t-\tau(w(t)))
\end{array}\right.
$$

with

$$
\begin{aligned}
\tau(w) & =\frac{L}{\Theta(w)}-K(w), \\
K(v) & =\sqrt{\frac{2 L E(v)}{\Theta(v)^{3}}},
\end{aligned}
$$

where $\Theta(v)$ and $E(v)$ are given by equations (2) and (3), the initial state given by $v_{0}(t)=Q(0, t)$, for $t \in\left[-\tau_{\max }, 0\right]$, where $\tau_{\max }$ is the maximum value of the delay, obtained in our case for the minimum value of the input discharge.

Then each linearization of (22) around an equilibrium trajectory $u_{0}=$ $v_{0}=Q_{0}$ coincides with the linear system (21) where $\lambda=Q_{0}$.

Indeed, the linearization of $(22)$ around $\left(v_{0}, u_{0}\right)$ leads to :

$$
\frac{\partial f}{\partial v}\left(v_{0}, u_{0}\right)=-\frac{1}{K\left(v_{0}\right)}+\left(u_{0}-v_{0}\right) \frac{K^{\prime}\left(v_{0}\right)}{K^{2}\left(v_{0}\right)} .
$$

Since $u_{0}=v_{0}$, we recover the expression (21) for $v_{0}=\lambda$. We also have:

$$
\frac{\partial f}{\partial u}\left(v_{0}, u_{0}\right)=\frac{1}{K\left(v_{0}\right)}
$$

and the other terms are obtained in the same way.

Remark: This model is only valid for slow variations of the state $v$. It is also an underlying hypothesis for the diffusive wave model. If the discharge varies very quickly, the celerity and the diffusion depending on the discharge would certainly give results different from physical observations. Indeed, the diffusive wave equation is obtained by neglecting inertia terms in the dynamic equation of Saint-Venant's model [23]. These inertia terms contain $\frac{\partial Q}{\partial t}$, which is not negligible if the discharge varies quickly. 


\section{Application of the Methodology to the Saint-Venant Equations}

We now show that the proposed methodology can be applied on the SaintVenant equations, a set of hyperbolic nonlinear conservation laws. The proposed approach is developed along the following steps: linearization of the equations around a steady flow regime, Laplace transform and cumulant matching method to obtain a family of first order linear systems with delay indexed by the reference flow, and derivation of the nonlinear model.

\subsection{Saint-Venant Equations}

The Saint-Venant equations are classically used to describe the shallow water flow dynamics in an open-channel. The equations are given by:

$$
\begin{aligned}
\frac{\partial A(x, t)}{\partial t}+\frac{\partial Q(x, t)}{\partial x} & =0, \\
\frac{\partial Q(x, t)}{\partial t}+\frac{\partial}{\partial x}\left[\frac{Q^{2}(x, t)}{A(x, t)}\right]+g A(x, t)\left(\frac{\partial Y(x, t)}{\partial x}+S_{f}(x, t)-S_{b}\right) & =0 .
\end{aligned}
$$

where $S_{b}(\mathrm{~m} / \mathrm{m})$ is the bottom slope, $A(x, t)\left(\mathrm{m}^{2}\right)$ is the wetted area, and $g$ $\left(\mathrm{m} / \mathrm{s}^{2}\right)$ is the gravitational acceleration.

The friction slope $S_{f}$ is modeled with the classical Manning formula [9]:

$$
S_{f}=\frac{Q^{2} n^{2}}{A^{2} R^{4 / 3}},
$$

with $n$ the Manning coefficient $\left(\mathrm{sm}^{-1 / 3}\right)$ and $R$ the hydraulic radius $(\mathrm{m})$, defined by $R=A / P$, where $P$ is the wetted perimeter (m).

The linearized Saint-Venant equations around a uniform steady flow are given by:

$$
\begin{aligned}
W_{0} \frac{\partial y}{\partial t}+\frac{\partial q}{\partial x} & =0 \\
\frac{\partial q}{\partial t}+2 V_{0} \frac{\partial q}{\partial x}+\left(C_{0}^{2}-V_{0}^{2}\right) W_{0} \frac{\partial y}{\partial x}+\frac{2 g S_{b}}{V_{0}} q-g W_{0}(1+\kappa) S_{b} y & =0 .
\end{aligned}
$$

where $q(x, t)$ and $y(x, t)$ are the deviations from the uniform steady state values $Q_{0}$ and $Y_{0}$, and

$$
\kappa=\frac{7}{3}-\frac{4 A_{0}}{3 W_{0} P_{0}} \frac{\partial P_{0}}{\partial Y}
$$

$C_{0}=\sqrt{g A_{0} / W_{0}}$ is the wave celerity, $V_{0}=Q_{0} / A_{0}$ the flow velocity, and $W_{0}$ is the top width. 


\subsection{Saint-Venant Transfer Function}

In the case of a semi-infinite channel, the Saint-Venant transfer function is given by (see Appendix B for details):

$$
\hat{q}(x, s)=e^{\lambda_{S V}(s) x} \hat{q}(0, s),
$$

where

$$
\lambda_{S V}(s)=\left(a s+b-\sqrt{c s^{2}+d s+b^{2}}\right),
$$

and

$$
\begin{aligned}
a & =\frac{F_{0}}{C_{0}\left(1-F_{0}^{2}\right)} \\
b & =\frac{(1+\kappa) W_{0} S_{b}}{2 A_{0}\left(1-F_{0}^{2}\right)} \\
c & =\frac{1}{C_{0}^{2}\left(1-F_{0}^{2}\right)^{2}} \\
d & =\frac{S_{b} W_{0}}{V_{0} A_{0}} \frac{\left(2+(\kappa-1) F_{0}^{2}\right)}{\left(1-F_{0}^{2}\right)^{2}}
\end{aligned}
$$

and $F_{0}=V_{0} / C_{0}$ is the Froude number for the equilibrium regime.

We now use the cumulant matching method to identify a first order model with delay that approximates this transfer function for low frequencies. A similar approach was used by [12], to identify a model of a fractional order.

\subsection{Cumulant Matching}

The cumulants of $e^{\lambda_{S V}(s) L}$ can be computed using Eq. (7). Indeed, the Taylor series development of $\lambda_{S V}(s)$ around $s=0$ is given by:

$$
\lambda_{S V}(s)=-\frac{2}{(1+\kappa) V_{0}} s+\frac{\left(4-(\kappa-1)^{2} F_{0}^{2}\right)}{g S_{b}(1+\kappa)^{3} F_{0}^{2}} s^{2}+o\left(s^{2}\right) .
$$

Therefore, the cumulants of the input-output Saint-Venant transfer function are easily obtained. Following the same approach as the one developed on the Hayami transfer function, the cumulant matching method applied to the Saint-Venant transfer function and a first order system with delay leads to:

$$
\begin{aligned}
\log G & =0 \\
\tau+K & =\frac{2 L}{(1+\kappa) V_{0}} \\
K^{2} & =\frac{2\left[4-(\kappa-1)^{2} F_{0}^{2}\right] L}{g S_{b}(1+\kappa)^{3} F_{0}^{2}} .
\end{aligned}
$$


Solving for the unknowns $G, K$ and $\tau$ :

$$
\begin{aligned}
G & =1, \\
\tau & =\frac{2 L}{(1+\kappa) V_{0}}-K, \\
K & =\sqrt{\frac{2\left[4-(\kappa-1)^{2} F_{0}^{2}\right] L}{g S_{b}(1+\kappa)^{3} F_{0}^{2}} .}
\end{aligned}
$$

In the general case, it is not straightforward to express the model parameters as functions of the discharge $Q_{0}$. However, in the wide rectangular case, we can express the water depth $Y_{0}$ as a function of $Q_{0}$ :

$$
Y_{0}=\frac{Q_{0}^{0.6} n^{0.6}}{W_{0}^{0.6} S_{b}^{0.3}}
$$

Finally the model parameters can be expressed explicitly as functions of $Q_{0}$ :

$$
\begin{aligned}
\tau & =\frac{3 L W_{0}^{0.4} n^{0.6}}{5 Q_{0}^{0.4} S_{b}^{0.3}}-K \\
K & =\sqrt{\frac{3}{125}} \sqrt{\frac{\left(9-4 F_{0}^{2}\right) L}{g S_{b} F_{0}^{2}}}
\end{aligned}
$$

with $F_{0}^{2}=\frac{Q_{0}^{0.2} S_{b}^{0.9}}{g n^{1.8} W_{0}^{0.2}}$, and where we replaced $\kappa$ by $7 / 3$ (wide rectangular approximation).

\subsection{Nonlinear Model}

With the cumulant matching method, we have obtained a family of linear DDEs indexed by the value of the reference discharge $Q_{0}$, that are low frequency approximations of the Saint-Venant transfer function. Following the same approach as detailed above, we obtain a nonlinear model of first order with delay:

$$
\left\{\begin{array}{l}
\dot{v}(t)=\frac{1}{K(v(t))}(u(t)-v(t)) \\
w(t)=v(t-\tau(w(t)))
\end{array}\right.
$$

with $u(t)=Q(0, t), w(t)=Q(L, t)$,

$$
\begin{aligned}
\tau(w) & =\frac{3 L W_{0}^{0.4} n^{0.6}}{5 w^{0.4} S_{b}^{0.3}}-K(w), \\
K(v) & =\sqrt{\frac{3}{125}} \sqrt{\frac{\left(9-4 F_{0}(v)^{2}\right) L}{g S_{b} F_{0}(v)^{2}}},
\end{aligned}
$$


and $F_{0}(v)^{2}=\frac{v^{0.2} S_{b}^{0.9}}{g n^{1.8} W_{0}^{0.2}}$.

This nonlinear delay-differential model is a low frequency approximation of the nonlinear Saint-Venant equations in the case of a semi-infinite wide rectangular channel.

\section{Analysis of the Nonlinear Delay Differential Model}

The nonlinear model with delay obtained is not a standard one, since the delay depends on the output of the system. We first give the conditions on $\tau$ such that the system is well-posed, and then study its mass conservation properties.

\subsection{Well-posedness}

The observation equation of the nonlinear system with delay (22) is wellposed if and only if

$$
\tau(w(t))<t
$$

which implies

$$
\frac{\mathrm{d}}{\mathrm{d} t} \tau(w(t))<1
$$

This can be written

$$
\dot{w}(t) \tau^{\prime}(w(t))<1
$$

where $\tau^{\prime}=\frac{\mathrm{d} \tau(w)}{\mathrm{d} w}$.

The condition (27) can be checked at each time $t$. The well-posedness is in this case related to the fact that no shocks are present in the system. Similar conditions arise in the case of nonlinear kinematic wave equation (see the discussion in [20]).

\subsection{Mass Conservation}

An important issue when dealing with nonlinear models of flow routing is the mass conservation: does the model correctly reproduce this important physical conservation law?

For an open-channel system, mass conservation is guaranteed if the following equality holds for all $\left(x_{1}, x_{2}, t_{1}, t_{2}\right)$ :

$$
\int_{x_{1}}^{x_{2}}\left[A\left(x, t_{2}\right)-A\left(x, t_{1}\right)\right] d x=\int_{t_{1}}^{t_{2}}\left[Q\left(x_{1}, t\right)-Q\left(x_{2}, t\right)\right] d t
$$


where $A$ and $Q$ are the cross-sectional area and the discharge respectively. Taking $x_{1}=0$ and $x_{2}=L$ yields the following, particular condition in $u$ and $w$ for all $\left(t_{1}, t_{2}\right)$ (remember that $u(t)=Q(0, t)$ and $\left.w(t)=Q(L, t)\right)$ :

$$
\begin{aligned}
\int_{0}^{L}\left[A\left(x, t_{2}\right)-A\left(x, t_{1}\right)\right] d x & =\int_{t_{1}}^{t_{2}}[Q(0, t)-Q(L, t)] d t \\
& =\int_{t_{1}}^{t_{2}}[u(t)-w(t)] d t
\end{aligned}
$$

When $t_{2}$ tends to $t_{1}$, Eq. (30) becomes equivalent to

$$
\frac{\partial S}{\partial t}=u(t)-w(t)
$$

where $S$ is the total volume of water stored in the reach $[0, L]$. The limit expression of (28) when $t_{2} \rightarrow t_{1}$ and $x_{2} \rightarrow x_{1}$ is

$$
\frac{\partial A}{\partial t}+\frac{\partial Q}{\partial x}=0
$$

Any conservation law in the form (31) is conservative, that is, it verifies (28) and the particular case (30).

Consider now the flow model (31), with $Q$ defined as follows :

$$
\begin{aligned}
Q & =Q(A, \phi(x)) \quad \forall(x, t), x \in(0, L] \\
\frac{\partial Q}{\partial t}\left(0^{+}, t\right) & =\frac{1}{K(Q(0, t))} \frac{\partial S(t-\tau)}{\partial t}
\end{aligned}
$$

where $\phi(x)$ is a vector containing all the parameters on which $Q$ locally depends (e.g. Manning's friction coefficient, the bed slope of the channel, etc.). The parameter vector $\phi$ is constant in time. The flow model (31-33) corresponds to a kinematic wave routing model over the reach $(0, L]$, with the point $x=0$ serving as a controlled storage tank (see Fig. 1).

The input discharge to the tank (at $x=0^{-}$) is given by $u(t)$, while the output discharge of the tank $v(t)$ at $x=0^{+}$is given by Eq. (33b). The discharge wave travels into the channel $(x \in(0, L])$ according to a kinematic wave approximation (Eqs. (32, 33a)). Although the discharge law (33) is discontinuous, the system (32-33) is conservative because it verifies Eq. (28) and no particular assumption needs to be made about the continuous character of $A$ and $Q$ in the calculation of the integrals in Eq. (28). Note that $S$, 


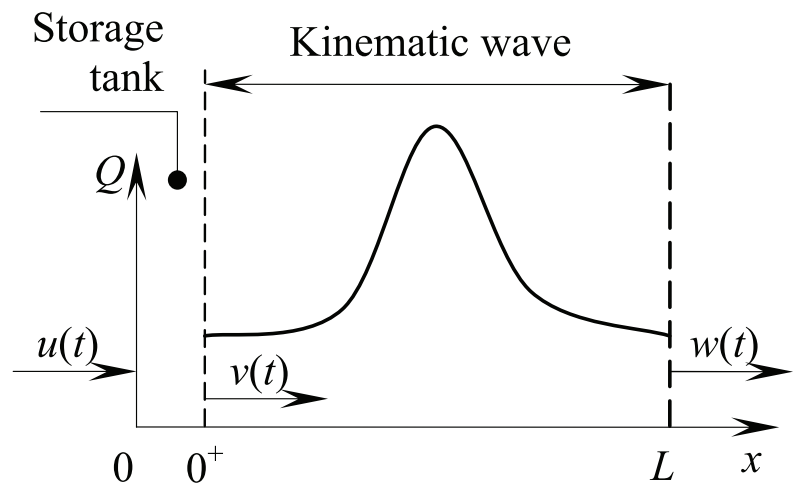

Figure 1: Conceptual representation of the model (32-33).

as defined in Eq. (31), includes not only the volume of water stored within the reach $(0, L]$ but also the volume of water present in the controlled storage tank.

The total volume of water stored in the reach $[0, L]$ is given by

$$
S=\int_{0}^{L}\left(A(x, t)+\delta(x) S_{0}(t)\right) d x=S_{0}(t)+\int_{0}^{L} A(x, t) d x
$$

where $\delta(x)$ is Dirac's distribution and $S_{0}(t)$ is the volume of water stored in the tank.

The characteristic form of Eqs. (32-33a) is known to be [11]

$$
\begin{aligned}
\frac{\mathrm{d} Q}{\mathrm{~d} t} & =0 \text { for } \frac{\mathrm{d} x}{\mathrm{~d} t}=\mu \\
\mu & =\frac{\partial Q}{\partial A}
\end{aligned}
$$

where $\mu$ is the propagation speed of the wave. Applying Eq. (34a) between $x=0^{+}$and $x=L$ yields

$$
Q(L, t)=Q\left(0^{+}, t-\tau\right),
$$

where $\tau$ is the travel time of the wave between $x=0^{+}$and $x=L$. Since there is a one-to-one relationship between $Q$ and $A$ at all points, $\mu$ depends only on $Q$ and $\tau$ is a function of $Q$ alone. Consequently, $\tau$ is a function of $w$. 
Noticing that $Q(0, t)=u(t), v(t)=Q\left(0^{+}, t\right)$ and $Q(L, t)=w(t)$, Eqs. (33b) and (35) become

$$
\begin{aligned}
\frac{\mathrm{d} v}{\mathrm{~d} t} & =\frac{1}{K}(u(t)-v(t)) \\
w(t) & =v(t-\tau)
\end{aligned}
$$

which is precisely the nonlinear model with delay (22). Since the model (3133 ) is conservative, the nonlinear model with delay (22) is also conservative.

We now compare the obtained simplified models to the original PDE models using simulated and real data.

\section{Applications of the Nonlinear Models}

We first perform simulations with the model derived based on the diffusive wave equation. Then, we test the model based on the Saint-Venant equations on real data.

The nonlinear system being composed of a nonlinear system with a delay in output, the nonlinear system without delay is first solved with a RungeKutta method of order 4, and the delayed output is computed in a second step.

\subsection{Nonlinear Model Simulations}

We consider a river of length $L=10 \mathrm{~km}$, width $W=8 \mathrm{~m}$, Manning coefficient $n=0.05$, slope $S_{b}=0.0004$. The parameters $K$ and $\tau$ of the nonlinear model can be computed as functions of the discharge $Q$ using eqs. $(14-15)$ and $(2-3)$. The results are depicted in Fig. 2. We see that the time delay $\tau$ varies between 204 min and 5 min when the discharge varies between $1 \mathrm{~m}^{3} / \mathrm{s}$ and $100 \mathrm{~m}^{3} / \mathrm{s}$, while the time constant $K$ varies between $58 \mathrm{~min}$ and 36 min.

These model parameters are used to compute the output of the simplified model given by Eq. (22). Validation is done by comparing the output of the proposed nonlinear model with that of the diffusive wave model (1) for the same input data. The diffusive wave equation (1) is solved using a semi-implicit finite difference scheme (Crank-Nicholson scheme). We also compare the output of the proposed model (22) with that given by the linear Hayami model obtained by linearization around the initial discharge $u(0)$. The simulation results are depicted in Fig. 3. 


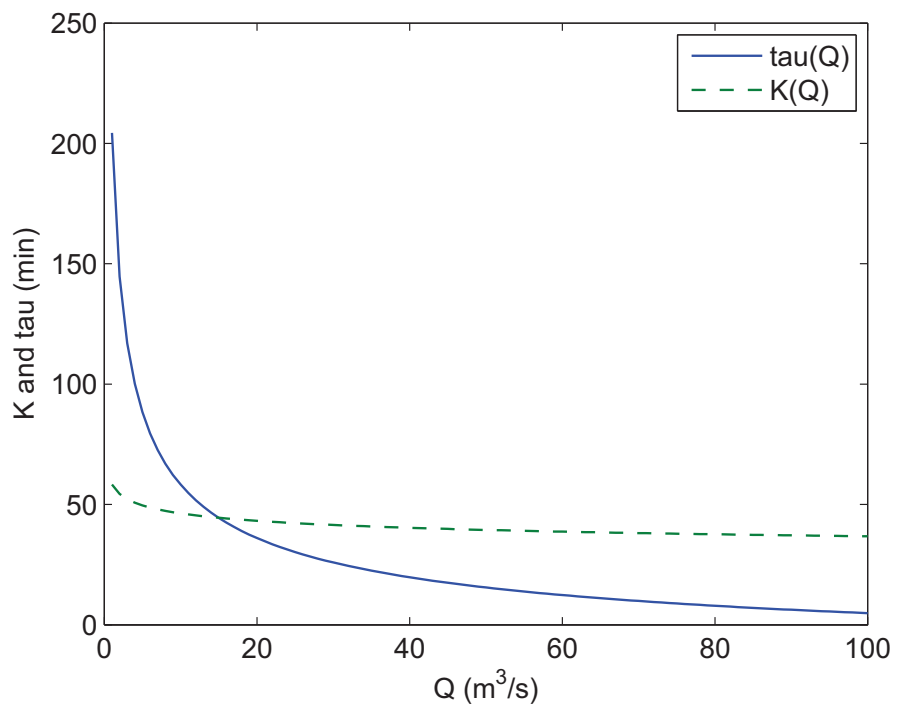

Figure 2: Values of parameters $K$ and $\tau$ as functions of $Q$

The nonlinear model with delay reproduces well the behavior of the river, and is very close to the diffusive wave model. One notices that the main characteristic of these rivers for low flows, i.e. the variation of the delay with the discharge, is taken well into account by the nonlinear model with delay, whereas this is not the case for the linear model.

\subsection{Comparison with Real Data}

We now test the simplified model (26) based on the linearized SaintVenant equations. To this end, we use the set of real data used by Baptista and Michel to evaluate the performance of the quadratic lag and route model [3]. This data consists of propagation of dam releases on the Jacui river in Brazil between Itauba and Volta Grande, recorded at a time step of 30 minutes [2].

The geometry of the river reaches is detailed in Table 1 . The downstream flow is computed by using a cascade of nonlinear models, one for each reach. The input of the first reach is the upstream flow, and the computed downstream flow is used as an input for the next reach. The computation is done for the five reaches in series, and only the downstream flow of the last reach is depicted in the graphical results. 

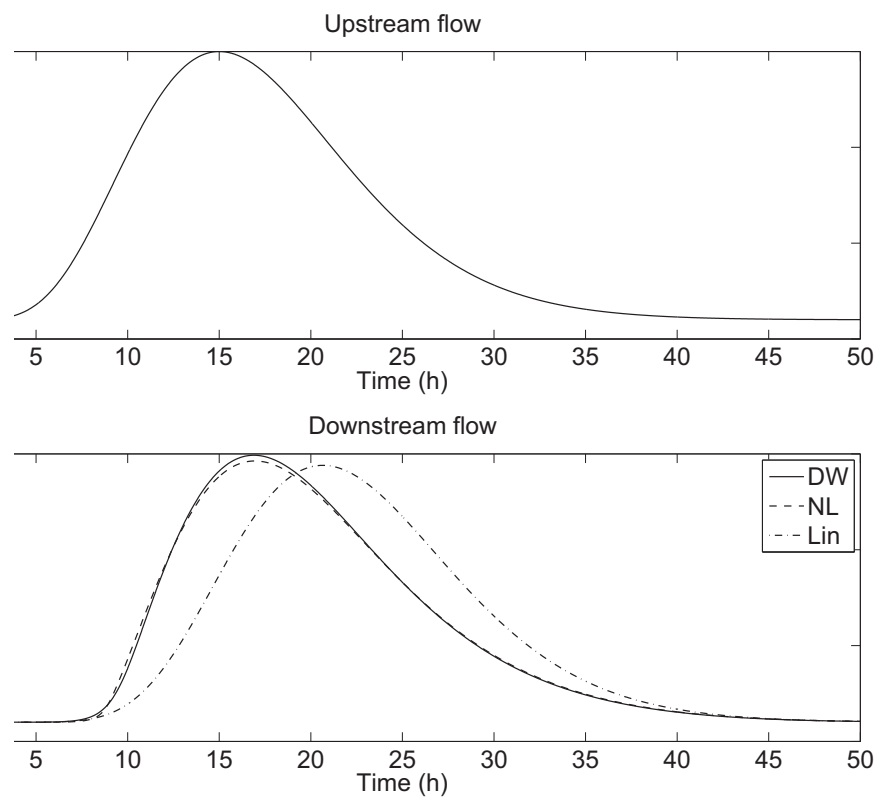

Figure 3: Comparison between the diffusive wave model (DW - ) , the nonlinear model of first order with delay (NL - -) and the linear Hayami model obtained for $Q_{0}=Q(0)(\operatorname{Lin}$ $-\cdot-)$

Simulation results are depicted in Fig. 4. The model only uses the data given in Table 1 and the upstream flow to compute the downstream flow. The Nash-Sutcliffe coefficient is equal to 0.91 , which is rather good. We also depict on the same graph the simulation results obtained with a full nonlinear Saint-Venant model, which also uses the geometry of the five reaches in series to compute the downstream flow. A uniform depth boundary condition is used at the downstream end.

The simplified nonlinear model leads to almost identical results as those obtained with the Saint-Venant equations, which explains why the two curves are indistinguishable. This shows that a simplified nonlinear model can efficiently reproduce the flow transfer described by the full Saint-Venant equations in this case. 
Table 1: Physical characteristics of the Jacui river reaches

\begin{tabular}{c|c|c|c|c}
\hline \hline Reach & $L$ & $W$ & $S_{b}$ & $n$ \\
\hline 1 & 3000 & 50 & 0.00169 & 0.07 \\
2 & 12600 & 60 & 0.00067 & 0.07 \\
3 & 3600 & 70 & 0.00194 & 0.07 \\
4 & 10400 & 80 & 0.00056 & 0.07 \\
\hline \hline
\end{tabular}

\subsection{Model Calibration}

From the simulation results in Fig. 4, it seems that the model leads to a larger attenuation than in reality, i.e. the time constant $K$ is too large. This could come from a bad calibration of the Manning coefficient, or a bad estimation of the geometry of the river reaches. To illustrate a possible application of the model, we now consider the river as a single reach, and try to identify physical parameters based on the input-output behavior of the system. To this end, we fix the length of the river reach $L=29600 \mathrm{~m}$ and the Manning coefficient $n=0.07$, and we identify an average geometry (width $B$ and slope $S_{b}$ ) by minimizing the sum of the quadratic error between the measured and the computed downstream flows.

The optimization resulted in an average width $B=55.6 \mathrm{~m}$ and an average slope $S_{b}=0.00089$. The Nash coefficient is then equal to 0.95 instead of 0.91 for the simulation case without calibration (see the simulation results in Fig. 5). This example illustrates a possible use of the model for parameter estimation. However, the analysis of the calibration of a model in the case of partially known or simplified geometry is out of the scope of this paper. This subject would require a more in depth study. This simple example is just here to show a possible application of the proposed simplified model.

\section{Conclusion}

We described in this paper a way to obtain a simple DDE nonlinear model from a quasilinear PDE. After linearization around a reference regime, the cumulant matching method is applied to the linear PDE in order to get an approximation of its irrational transfer function. Then a single nonlinear model is obtained from the family of linear ones indexed by the value of the discharge around which they were linearized. This methodology was applied on two different nonlinear PDEs: the diffusive wave equation and the Saint- 

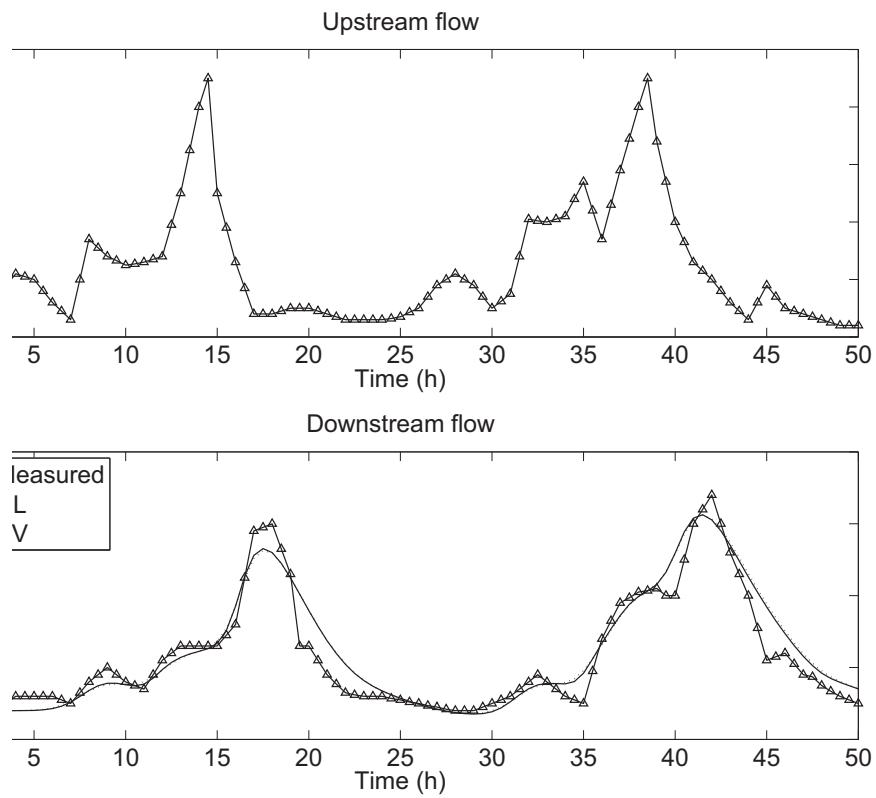

Figure 4: Simulation on real data from the Jacui river. Measured upstream flow $(-\triangle-)$, measured downstream flow $(-\triangle-)$, downstream flow simulated by the nonlinear model $(-)$ and by the Saint-Venant equations $(--)$

Venant equations. A first order nonlinear model with delay is obtained as an approximation of the nonlinear PDE.

The simplified nonlinear model has the advantage to be directly related to physical parameters, as the length of the river stretch $L$, the slope $S_{b}$, Manning coefficient $n$ and the width $W$. This model can be used for many different purposes:

- For quick simulation purposes, as it is easier to implement than a complete numerical resolution of the initial partial differential equation,

- For identification purposes: a model-based identification usually requires numerous simulations of the model, which is time-consuming. A simpler model can be simulated more quickly,

- For controller design, as some dams located upstream can be used to control the discharge downstream of the river (see e.g. $[19,21,6]$ ). 


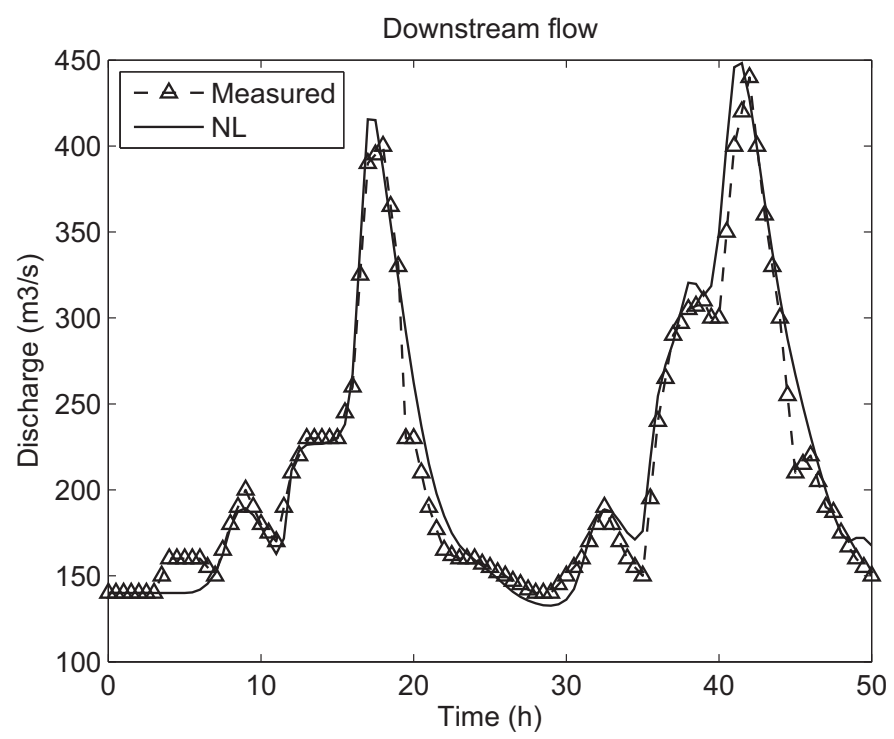

Figure 5: Calibration of the simplified nonlinear model on real data from the Jacui river. Measured downstream flow $(-\triangle-)$ and downstream flow simulated by the nonlinear model $(-)$.

Finally, the proposed approach can be generalized to various other cases, such as the model of first order with delay derived by [25] for rivers or canals with a backwater curve. The proposed method provides a rigorous way to derive a nonlinear model from a set of linear models, which can prove very useful in the hydrological context.

\section{Acknowledgements}

The help of Julien Lerat, Charles Perrin and Marció Baptista who provided the data of the Jacui river is gratefully acknowledged.

\section{A. Derivation of the Hayami Transfer Function}

The linear partial differential equation (4) has an analytical solution for simple inputs (steps or ramps) (see e.g. [24]). Using Laplace transform, one can express the input-output relations as a transfer function [1]. Given a function $f(x, t), t \geq 0$, its temporal Laplace transform, denoted $\mathcal{L}(f(x, t))$, 
or $\hat{f}(x, s)$ is given by:

$$
\mathcal{L}(f(x, t))=\hat{f}(x, s)=\int_{0}^{\infty} e^{-s t} f(x, t) \mathrm{d} t .
$$

We then have:

$$
\mathcal{L}\left(\frac{\partial f(x, t)}{\partial t}\right)=s \hat{f}(x, s)-f(x, 0)
$$

and

$$
\mathcal{L}\left(\frac{\partial f(x, t)}{\partial x}\right)=\frac{\partial \hat{f}(x, s)}{\partial x}
$$

and

$$
\mathcal{L}\left(\frac{\partial^{2} f(x, t)}{\partial x^{2}}\right)=\frac{\partial^{2} \hat{f}(x, s)}{\partial x^{2}} .
$$

Applying Laplace transform to the system (4) leads to:

$$
E_{0} \frac{\partial^{2} \hat{q}(x, s)}{\partial x^{2}}-\Theta_{0} \frac{\partial \hat{q}(x, s)}{\partial x}-s \hat{q}(x, s)=q(x, 0) .
$$

This is in fact an ordinary differential equation in the variable $x$, whose characteristic equation

$$
E_{0} \lambda^{2}-\Theta_{0} \lambda-s=0
$$

has two solutions if $E_{0} \neq 0$.

With the hypothesis $q(x, 0)=0$, the solution $\hat{q}(x, s)$ is of the following form:

$$
\hat{q}(x, s)=A_{1}(s) e^{\lambda_{1}(s) x}+A_{2}(s) e^{\lambda_{2}(s) x},
$$

with

$$
\begin{aligned}
& \lambda_{1}(s)=\frac{\Theta_{0}-\sqrt{\Theta_{0}^{2}+4 E_{0} s}}{2 E_{0}}, \\
& \lambda_{2}(s)=\frac{\Theta_{0}+\sqrt{\Theta_{0}^{2}+4 E_{0} s}}{2 E_{0}} .
\end{aligned}
$$

The boundary conditions can now be used to specify variables $A_{1}(s)$ and $A_{2}(s)$. The downstream boundary condition leads to $A_{2}(s)=0$, since $\Re\left(\lambda_{2}(s)\right)>0$ for $\Re(s)>0$. The upstream boundary condition leads to $A_{1}(s)=\hat{q}(0, s)$. Finally, the Hayami transfer function is obtained as follows:

$$
\hat{q}(x, s)=F_{\text {Hayami }}(x, s) \hat{q}(0, s)
$$

with

$$
F_{\text {Hayami }}(x, s)=e^{\frac{\Theta_{0}-\sqrt{\Theta_{0}^{2}+4 E_{0} s}}{2 E_{0}} x} .
$$




\section{B. Derivation of the Saint-Venant Transfer Function}

Following the approach developed by $[17,16]$, the Saint-Venant transfer function can be computed based on the linearized equations. Applying Laplace transform to Eqs. (24), and assuming zero initial conditions leads to:

$$
\frac{\partial}{\partial x}\left(\begin{array}{l}
\hat{q} \\
\hat{y}
\end{array}\right)=\mathcal{A}_{s}\left(\begin{array}{l}
\hat{q} \\
\hat{y}
\end{array}\right)
$$

with $\mathcal{A}_{s}=\left(\begin{array}{cc}0 & -W_{0} s \\ \frac{2 g S_{b}-V_{0} s}{W_{0} V_{0} C_{0}^{2}\left(1-F_{0}^{2}\right)} & \frac{2 V_{0} s+g(1+\kappa) S_{b}}{C_{0}^{2}\left(1-F_{0}^{2}\right)}\end{array}\right)$.

Matrix $\mathcal{A}_{s}$ has two eigenvalues, given by:

$$
\lambda(s)=a s+b \pm \sqrt{c s^{2}+d s+b^{2}},
$$

with $a, b, c$ and $d$ given by Eqs. (25).

Assuming a semi-infinite channel leads to:

$$
\hat{q}(x, s)=e^{\lambda_{S V}(s) x} \hat{q}(0, s)
$$

with $\lambda_{S V}(s)=a s+b-\sqrt{c s^{2}+d s+b^{2}}$.

\section{References}

[1] A. Angot. Compléments de mathématiques à l'usage des ingénieurs de l'électrotechnique et des télécommunications. Masson et Cie, Paris, 6th edition, 1972. in French.

[2] M. Baptista. Contribution à l'étude de la propagation de crues en hydrologie. PhD thesis, Ecole Nationale des Ponts et Chaussées, Paris, Septembre 1990.

[3] M. Baptista and C. Michel. Influence des caractéristiques hydrauliques des biefs sur la propagation des pointes de crue. La Houille Blanche, 2:141-148, 1990 .

[4] J.-P. Baume, J. Sau, and P.-O. Malaterre. Modeling of irrigation channel dynamics for controller design. In Conf. on Systems, Man and Cybernetics, SMC'98, pages 3856-3861, San Diego, 1998. 
[5] P.L.F. Bentura and C. Michel. Flood routing in a wide channel with a quadratic lag-and-route method. Hydrological Sciences, 42(2):169-189, 1997.

[6] G. Besançon, D. Georges, and Z. Benayache. Towards nonlinear delaybased control for convection-like distributed systems: the example of water flow control in open channel systems. Networks Heterogeneous Media, 4(2):211-222, 2009.

[7] K.J. Beven. Prophecy, reality and uncertainty in distributed hydrological modelling. Adv. Water Resourc., 16:41-51, 1993.

[8] L.A. Camacho and M.J. Lees. Multilinear discrete lag-cascade model for channel routing. J. of Hydrology, 226:30-47, 1999.

[9] V.T. Chow. Open-channel hydraulics. McGraw-Hill, New York, 1988. 680 p.

[10] J.A. Cunge. On the subject of a flood propagation computation method (Muskingum method). J. Hydr. Res., 7(2):205-230, 1969.

[11] J.A. Cunge, F.M. Holly, and A. Verwey. Practical aspects of computational river hydraulics. Pitman Advanced Publishing Program, 1980.

[12] J.C.I. Dooge, J.J. Napiórkowski, and W.G. Strupczewski. The linear downstream response of a generalized uniform channel. Acta Geophys. Polon., XXXV(3):279-293, 1987.

[13] S. Hayami. On the propagation of flood waves. report, Disaster Prevention Institute, University of Kyoto, 1951.

[14] M. Lal and R. Mitra. A comparison of transfer function simplification methods. IEEE Trans. Autom. Contr., 19(5):617-618, 1974.

[15] X. Litrico. Nonlinear diffusive wave modeling and identification for openchannels. J. Hydraul. Eng., 127(4):313-320, 2001.

[16] X. Litrico and V. Fromion. Frequency modeling of open channel flow. J. Hydraul. Eng., 130(8):806-815, 2004.

[17] X. Litrico and V. Fromion. Modeling and control of hydrosystems. Springer, London, 2009. 
[18] X. Litrico and D. Georges. Robust continuous-time and discrete-time flow control of a dam-river system (I): Modelling. Appl. Math. Mod., 23(11):809-827, 1999.

[19] X. Litrico and D. Georges. Robust continuous-time and discrete-time flow control of a dam-river system (II): Controller design. Appl. Math. Mod., 23(11):829-846, 1999.

[20] X. Litrico and V. Guinot. Discussion of "Development and Verification of an Analytical Solution for Forecasting Nonlinear Kinematic Flood Waves" by Sergio E. Serrano, Journal of Hydrologic Engineering, July/August 2006, Vol. 11, No. 4, pp. 347-353. J. of Hydrologic Engineering, 12(6):703-705, 2007.

[21] X. Litrico and J.-B. Pomet. Nonlinear modeling and control of a long river stretch. In European Control Conference, Cambridge, UK, 2003.

[22] P.-O. Malaterre. Modélisation, analyse et commande optimale LQR d'un canal d'irrigation. PhD thesis, ENGREF-Cemagref, 1994. (in French).

[23] W.A. Miller and J.A. Cunge. Simplified equations of unsteady flow, pages 183-257. Water Resources Publications, Fort Collins, CO, 1975.

[24] R. Moussa and C. Bocquillon. Criteria for the choice of flood routing methods in natural channels. J. of Hydrology, 186(1-4):1-30, 1996.

[25] S. Munier, X. Litrico, G. Belaud, and P.-O. Malaterre. Distributed approximation of open-channel flow routing accounting for backwater effects. Adv. Water Resourc., 31:1590-1602, 2008.

[26] M. Perumal, T. Moramarco, and F. Melone. A caution about the multilinear discrete lag-cascade model for flood routing. J. of Hydrology, 338(3-4):308-314, May 2007.

[27] V.M. Ponce and F.D. Theurer. Accuracy criteria in diffusion routing. J. of Hydraulics Division, 108(HY6):747-757, 1982. 